\title{
Estudio entre las tecnologías WIFI - LIFI en la optimización del servicio de internet
}

\section{Study between WIFI - LIFI technologies in the optimization of internet service}

\author{
Oscar Efrén Cárdenas Villavicencio ${ }^{1, *}$, Jimmy Rolando Molina Ríos ${ }^{1, \dagger}$, \\ Rodrigo Fernando Morocho Román ${ }^{1, \ddagger}$, Johnny Paúl Novillo Vicuña ${ }^{1, \otimes}$, y \\ Galo Rodrigo Moreno Sotomayor ${ }^{1, \diamond}$ \\ ${ }^{1}$ Universidad Técnica de Machala, Ecuador. \\ oecardenas@utmachala.edu.ec,jmolina@utmachala.edu.ec,rmorocho@utmachala.edu.ec, \\ jnovillo@utmachala.edu.ec,gmoreno@utmachala.edu.ec \\ Fecha de recepción: 03 de octubre de 2017 - Fecha de aceptación: 23 de noviembre de 2017 \\ DOI: https://doi.org/10.26910/issn.2528-8083vol2iss8.2017pp50-53
}

\begin{abstract}
Resumen-En la presente investigación realizaremos una comparación entre las tecnologías WI-FI y LI- FI, en la cual determinaremos las ventajas y desventajas que presentan estas tecnologías al momento de ser implementadas. Emplearemos un método comparativo en donde analizaremos características como la forma de transmisión, la velocidad y el alcance de la señal de ambas tecnologías. Según como avance la investigación entre estas dos tecnologías inalámbricas podremos determinar cuál de estas nos presta los mejores servicios en términos de transmisión y seguridad. Al finalizar la investigación podremos indicar cuál de estas tecnologías es la ideal de emplear en la mejora del servicio de internet. Concluimos que ambas tecnologías nos pueden ofrecer un buen servicio de internet, sin embargo, la tecnología LI-FI es la opción más conveniente de implementar porque nos brinda una gran velocidad y seguridad en la transmisión de la información en comparación al empleo de la tecnología WI-FI la cual brinda una velocidad y seguridad menor.
\end{abstract}

Palabras Clave-WIFI, LIFI, Internet.

Abstract-In the present investigation, we will make a comparison between the WI-FI and LI-FI technologies, in which we will determine the advantages and disadvantages of these technologies when they are implemented. We will use a comparative method where we will analyze characteristics such as the transmission method, the speed and the signal range of both technologies. According to how the research between these two wireless technologies advances, we will be able to determine which of these provides the best services in terms of transmission and security. At the end of the investigation we can indicate which of these technologies is the ideal to use in improving the internet service. We conclude that both technologies can offer us a good internet service, however, the LI-FI technology is the most convenient option to implement because it gives us great speed and security in the transmission of information compared to the use of WI technology. -FI which provides a lower speed and safety.

Keywords-WIFI, LIFI, Internet.

\section{INTRODUCCIÓN}

$\mathbf{L}$ as tecnologías inalámbricas han mejorado la forma de transmitir la información, permitiendo él envió y la recepción de datos sin ser necesaria la conexión de nuestros dispositivos con cables. La transmisión sin cables permite agilizar las conexiones en una red, proporcionando un rápido acceso a la información en cualquier sitio en donde esté ubicada la red.

La tecnología WI-FI es un sistema que emplea ondas de radio como medio para transmitir y receptar información entre otros dispositivos sin la utilización de ningún cable. El WIFI está presente en casi todos los dispositivos electrónicos como teléfonos y computadores, permitiendo tener una rápida conexión de estos al internet (Armijos De La Vera et al., 2015).

\footnotetext{
*Ingeniero de Sistemas, Magister en Telecomunicaciones,

${ }^{\dagger}$ Ingeniero de Sistemas, Magister en Docencia y Gerencia en Educación Superior

${ }^{\ddagger}$ Ingeniero de Sistemas, Magister en Seguridad Informática Aplicada

${ }^{\otimes}$ Ingeniero Eléctrico, Magister en Educación Superior

${ }^{\diamond}$ Economista, Magister en Administración de Empresas
}

El gran uso de esta tecnología en nuestros dispositivos, ha originado que lugares públicos como terminales, parques, centros comerciales entre otros; ofrezcan el servicio de internet utilizando redes WI-FI, las cuales permiten conectarse y navegar rápidamente.

La tecnología LI-FI es una nueva forma de transmitir la información inalámbricamente utilizando la luz para la transmisión de los datos. Utiliza la luz generada por los LED como lámparas y focos para enviar la información y es receptada por un foto sensor instalado en los equipos con tecnología LI-FI (Bermeo and Fajardo, 2013).

WI-FI El WI-FI (Abreviación de Wireless Fidelity) es un sistema de comunicacion inalámbrico que permite la conexion entre dispositivos sin utilizar ningun cable. Emplea las ondas de radio para la transmisión y la recepción de los datos (Armijos De La Vera et al., 2015).

Elementos de un sistema WI-FI

1. Punto de acceso (AP): Es el dispositivo que permite la 
conexión de los equipos inalámbricos a la red.

2. Clientes WI-FI: Son los dispositivos equipados con tarjetas WI-FI como ordenadores y teléfonos.

3. SSID: Es el nombre que identifica a la red WI-FI. Agrupa a todos las conexiones que están en la red.

4. Roaming: EPermite a los usuarios tener movilidad e ir alternando de punto acceso dependiendo de la intensidad de la señal.

\section{Funcionamiento del WI-FI}

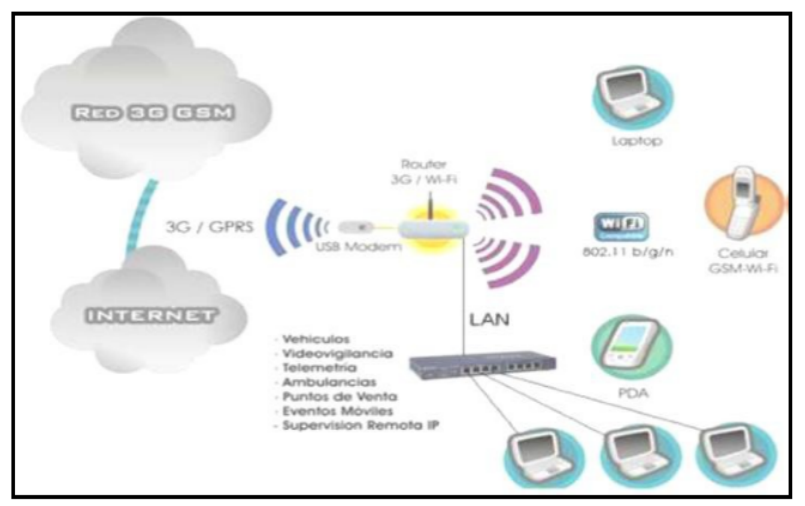

Figura 1. Funcionamiento del WI-FI

Fuente: http://www.redesinternet.com/que-es-una-conexion-wifi-ii/

Un router se conecta por un cable directamente a un modem o al dispositivo que provee el servicio de internet, este router se encarga de enviar los datos que recibe a través de las ondas de radio que son transmitidas en el toda el área de alcance del router. Estas ondas son receptadas por nuestros dispositivos WI-FI, los cuales tienen un software que se encarga de decodificarlas e interpretarlas para su uso.

\section{Estándar}

Las redes y dispositivos WI-FI emplean el estándar IEEE 802.11, el cual fue desarrollado con para la comunicación de dispositivos inalámbricos. Este estándar determina las capas del modelo OSI para un enlace inalámbrico utilizando ondas electromagnéticas (IEEE, 2012).

El estándar IEEE 802.11 se subdivide en:

- IEEE 802.11b

- IEEE 802.11a

- IEEE $802.11 \mathrm{~g}$

- IEEE 802.11n

El estándar IEEE 802.11 determina dos modos de funcionamiento:

\section{- Modo Infraestructura:}

Este modo es el usado en las mayorías de redes WI-FI, el cual utiliza un punto de acceso que es conectado a una parte de la red utilizando un cable de tipo coaxial o UTP, a través de este punto se conectan los ordenadores a la red.

- Modo Ad Hoc:

Este modo permite conectar los ordenadores directamente entre ellos, sin emplear un punto de acceso. Es conocido como redes de punto a punto.

\section{Transmisión}

El WI-FI transmite la informacion utilizando las ondas de radio, estas ondas son muy propensas a ser afectadas por diversos tipos de interferencias como son:

- La reflexión de la señal.

- Condiciones climáticas.

- Materiales que impiden el paso de la señal.

- Señales producidas por distintos dispositivos de comunicación.

El nuevo estándar 802.11n emplea dispositivos WI-FI con varias antenas internas para permitir transmitir y receptar señal por diversas rutas logrando que el sistema sea más estable ante las interferencias. Además, utiliza 2 canales de transmisión con un ancho de banda de 20 y $40 \mathrm{MHz}$ lo cual otorga a la red una mayor velocidad (Escudero, 2012).

Tabla 1. Parámetros de transmisión del WI-FI

\begin{tabular}{|c|c|c|c|c|}
\hline ESTANDAR & $\mathbf{8 0 2 . 1 1 a}$ & $802.11 \mathrm{~b}$ & $802.11 \mathrm{~g}$ & $\mathbf{8 0 2 . 1 1 n}$ \\
\hline Frecuencia & $5 \mathrm{GHz}$ & $2.4 \mathrm{GHz}$ & $2.4 \mathrm{GHz}$ & $\begin{array}{l}2.4 \mathrm{GHz} \\
\text { y } 5 \mathrm{GHz}\end{array}$ \\
\hline Ancho de,banda & $20 \mathrm{MHz}$ & $20 \mathrm{MHz}$ & $40 \mathrm{MHz}$ & $\begin{array}{l}20 \mathrm{MHz} \\
\text { y } 40 \mathrm{MHz}\end{array}$ \\
\hline \multicolumn{5}{|c}{ Fuente: Elaboración Propia. }
\end{tabular}

\section{Distancia}

La distancia de la señal depende del alcance que ofrescan dispositivos WI-FI para la transmisión de la señal y de los diversos materiales por los cuales la señal tendrá que pasar, por ejemplo: las paredes. Los diversos estándares del WI-FI nos indican las siguientes distancias que puede tomar la señal.

Tabla 2. Distancia de la señal WI-FI

\begin{tabular}{|c|l|l|l|l|}
\hline ESTANDAR & \multicolumn{1}{|c|}{ 802.11a } & $802.11 \mathrm{~b}$ & $802.11 \mathrm{~g}$ & $\mathbf{8 0 2 . 1 1 n}$ \\
\hline \multirow{2}{*}{ Distancia } & $\begin{array}{l}\text { Entre 10 a,30 } \\
\text { metros }\end{array}$ & $\begin{array}{l}90 \\
\text { metros }\end{array}$ & $\begin{array}{l}50 \\
\text { metros }\end{array}$ & $\begin{array}{l}20 \\
\text { a 30 metros }\end{array}$ \\
\hline
\end{tabular}

Fuente: Elaboración Propia.

\section{Velocidad}

La velocidad en una red WI-FI depende del bando de ancho empleado en la transmisión de los datos y de la distancia existente entre los dispositivos conectados.

Tabla 3. Velocidad de la señal WI-FI

\begin{tabular}{|c|l|l|l|l|}
\hline ESTANDAR & 802.11a & $802.11 \mathrm{~b}$ & $802.11 \mathrm{~g}$ & \multicolumn{1}{|c|}{$\mathbf{8 0 2 . 1 1 n}$} \\
\hline Velocidad & 54 & 80 & 130 & $\begin{array}{l}\text { De } \\
\text { Mb Mbps } \\
\text { hasta 300 Mbps }\end{array}$ \\
\hline
\end{tabular}

Fuente: Elaboración Propia.

\section{Seguridad}

La seguridad de una red WI-FI es muy importante debido a que la señal WI-FI se propaga libremente en el espacio, la hace vulnerable a ser hackeada si no se emplean las medidas de seguridad correspondientes (Barajas Belmonte, 2016).

Los principales sistemas de seguridad de una red WI-FI son: 


\section{WEP}

Permite cifrar la red con una clave entre 40 y 104 bits. El WEP no presta una buena seguridad y no es recomendable utilizarlo.

\section{WPA}

Fue creado como un sustituto al WEP, cubría las principales falencias de seguridad de su antecesor. Permite utilizar claves de inicio de sesión de 128 bits para cada usuario de la red.

\section{WPA2}

Es una mejora del WPA, igual que el anterior cada usuario posee una clave para acceder a la red.

\section{Filtrados Mac}

Indica al punto de acceso (AP) que solo permita la conexión a los dispositivos con la dirección MAC (Media Access Control) especificadas.

\section{Ventajas y desventajas del WI-FI Ventajas}

- Permite tener un acceso rápido y fácil al internet.

- Instalar una red WI-FI es más rápido en comparación a la instalación de una red cableada.

- Permite la conexión en cualquier sitio en donde tenga alcance la red logrando así tener movilidad.

- Permite extender una red cableada a lugares en donde esta no tenga alcance.

- Conectar un nuevo ordenador a la red es muy fácil y no requiere de mucha configuración.

- El WI-FI es una tecnología muy comercializada por lo que es fácil de encontrar los equipos necesarios para armar una red.

\section{Desventajas}

- La velocidad y la transmisión del WI-FI es menor en comparación a una red cableada.

- La señal puede ser afectada por interferencias causadas por el clima u otros tipos de fenómenos.

- La señal WI-FI se esparce libremente ocasionando que personas desconocidas puedan hackearla y acceder a la información.

- No puede ser empleada en determinados sitios en los cuales las radiofrecuencias ocasionen interferencias como en hospitales y aviones.

- Puede ocasionar problemas con otros dispositivos de comunicación debido al uso del mismo espectro radioeléctrico.

\section{LI-FI}

Es un sistema de comunicación inalámbrica por el cual se puede transmitir y receptar información utilzando la luz. Esta tecnología emplea la luz genera por diodos LED (focos, lámparas) para enviar los datos y los recepta a través de un sensor que detecta los cambios de la luz.

"Es una tecnologia nueva desarrollada por el fisico Harald Haas de la Universidad de Edimburgo y la presento en el
2010 durante un conferencia en la cual realizo un experimento utilizando una linterna LED para enviar datos a 10 Mbps a un ordenador" (Bermeo and Fajardo, 2013).

\section{Funcionamiento de la tecnología LI-FI}

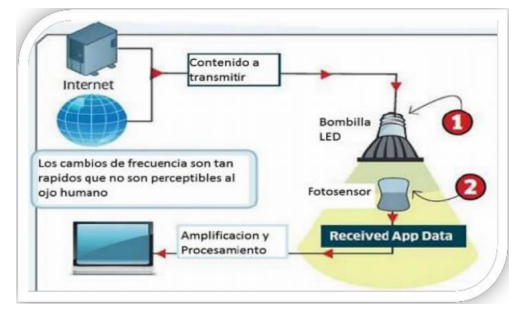

Figura 2. Funcionamiento del LI-FI

Fuente:

http://profesores.elo.utfsm.cl/ãgv/elo322/1s13/project/reports/Desafios _de_las_nuevas_tecnologias.pdf

El servidor o el dispositivo que provee internet son conectados a la red eléctrica para transmitir los datos.

Estos datos viajan a alta velocidad desde la lámpara LED la cual posee un modular que transforma los pulsos eléctricos en pulsos de luz. La luz emitida por el LED se apaga y se prende miles veces por segundo, generando una señal binaria (0 y 1) que es detectada por un fotosensor para luego ser decodificada y procesada por un software de datos digitales instalado en los dispositivos LI-FI.

Estándar: El estándar IEEE 802.15.7 es utilizado para la transmisión de información inalámbricamente empleando las frecuencias de luz visibles. Este estándar otorga una gran velocidad de transmisión que permite compartir información de un gran tamaño en pocos segundos (Tamayo Balas, 2016).

Transmisión: La tecnología LIFI como se ha señalado antes hace uso de la luz generada por los LED. En cada LED se coloca un dispositivo que actua como una antena y se encarga de enviar los datos a travez de la onda de luz generada. Esta onda es recibida por el sensor de los dispositivos LI-FI ubicados en el sitio de iluminacion.

La señal transmitida tiene una frecuencia de $385-789 \mathrm{THz}$ y posee un bando de ancho ilimitado, debido a que hace uso del espectro de luz que no usa licencia y es libre.

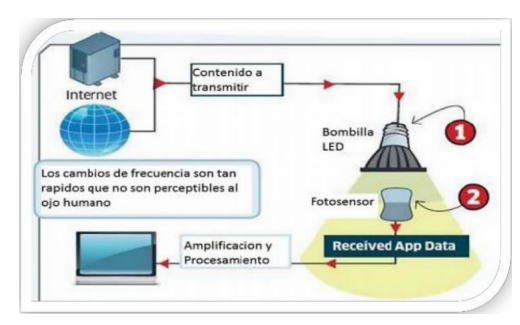

Figura 3. Transmisión de la señal LI-FI.

Fuente: http://purelifi.co.uk/wpcontent/uploads/2013/08/How_VLCworks.png 
Distancia: La distancia que tome la señal LI-FI depende de la onda de luz generada por el LED. La onda tiene un alcance corto y no atraviesa las paredes, originando que la señal quede limitada a una determinada área de iluminación.

Velocidad: La velocidad que nos puede otorgar el LI-FI según el último estándar es de $5 \mathrm{Gbps}$, una velocidad inmensa comparada con la velocidad proporcionada por otras tecnologías inalámbricas. La luz otorga una gran banda ancha y no sufre de interferencias que causan la disminución de la velocidad en otras tecnologías (Usma Oviedo et al., 2011).

Seguridad: La característica de la luz en no poder atravesar las paredes otorga una gran seguridad a la información a diferencia del WI-FI donde la señal se esparce libremente.

Para transmitir la información el emisor y receptor deben estar ubicados bajo un mismo sitio de iluminación, con lo cual podemos asegurar con quien estamos compartiendo la información.

\section{Ventajas y desventajas del LI-FI \\ Ventajas}

- Otorga una velocidad de transmisión mayor que otras tecnologías inalámbricas.

- No ocasiona interferencias como otras tecnologías inalámbricas porque usa el espectro de luz en vez de la radiofrecuencia.

- Como la luz no atraviesa las paredes se logra limitar la señal a un determinado sitio de iluminación por lo tanto la información tiene más seguridad.

- Puede adaptarse a cualquier fuente de luz instalando solamente un emisor LI-FI.

- Puede utilizarse en lugares en los cuales no es posible usar el WI-FI como hospitales.

- Permite compartir informacion de gran tamaño en pocos segundos.

\section{Desventajas}

- Tiene poco alcance obligando a utilizar varios emisores LI-FI para tener una red.

- Es necesario tener la iluminación siempre encendida para la transmisión de datos.

- Solo funciona con dispositivos que sean compatibles con esta tecnología.

- El LI-FI aun es una tecnología en desarrollo y no es comercializada masivamente.

- No funciona en lugares que están expuestos directamente a la luz solar los equipos necesarios para implementar una red LI-FI son muy costosos.

\section{Conclusión}

Al finalizar la investigación y analizar las características de ambas tecnologías inalámbricas determinamos que la ideal para optimizar el servicio de internet es el LI-FI, la cual nos ofrece grandes velocidades de transmisión para una excelente conexión a internet y mucha seguridad a la información, sin embargo, esta tecnología aun no es comercializada masivamente por lo tanto los elementos necesarios para su implementación son difíciles de conseguir además de tener un gran costo. En cambio, el WI-FI es un sistema muy demandado y su costo de implementación es más barato ofreciendo una buena velocidad para una conexión a internet.

\section{REFERENCIAS BIBLIOGRÁFICAS}

Armijos De La Vera, E. E., Bermudez, P., and Estefania, G. (2015). Estudio, análisis y optimización del tráfico de las redes wifi en la facultad de ingeniería en electricidad y computación.

Barajas Belmonte, A. (2016). Seguridad de redes de telecomunicaciones en el PREP del IEEM.

Bermeo, S. and Fajardo, C. (2013). Tecnología LI-FI. Universidad politécnica Salesiana-Sede Cuenca.

Escudero, A. (2012). Estándares en Tecnologías Inalámbricas. Obtenido de Universidad de Cataluña.

Tamayo Balas, A. (2016). Estudio del estándar 802.15. 7 del ieee sobre sistemas de comunicación por luz visible.

Usma Oviedo, J. S., Trujillo González, F., et al. (2011). Biodiversidad del departamento de casanare: identificación de ecosistemas estratégicos. 